University of Nebraska - Lincoln

DigitalCommons@University of Nebraska - Lincoln

$5-5-2003$

\title{
Spatial inhomogeneity of imprint and switching behavior in ferroelectric capacitors
}

Alexei Gruverman

University of Nebraska-Lincoln, agruverman2@unl.edu

B. J. Rodriguez

North Carolina State University, Raleigh, brian.rodriguez@ucd.ie

A. I. Kingon

North Carolina State University, Raleigh, angus_kingon@brown.edu

R. J. Nemanich

North Carolina State University, Raleigh

J. S. Cross

Fujitsu Laboratories, Atsugi 243-0197, Japan

See next page for additional authors

Follow this and additional works at: https://digitalcommons.unl.edu/physicsgruverman

Part of the Physics Commons

Gruverman, Alexei; Rodriguez, B. J.; Kingon, A. I.; Nemanich, R. J.; Cross, J. S.; and Tsukada, M., "Spatial inhomogeneity of imprint and switching behavior in ferroelectric capacitors" (2003). Alexei Gruverman Publications. 12.

https://digitalcommons.unl.edu/physicsgruverman/12

This Article is brought to you for free and open access by the Research Papers in Physics and Astronomy at DigitalCommons@University of Nebraska - Lincoln. It has been accepted for inclusion in Alexei Gruverman Publications by an authorized administrator of DigitalCommons@University of Nebraska - Lincoln. 


\section{Authors}

Alexei Gruverman, B. J. Rodriguez, A. I. Kingon, R. J. Nemanich, J. S. Cross, and M. Tsukada 


\title{
Spatial inhomogeneity of imprint and switching behavior in ferroelectric capacitors
}

\author{
A. Gruverman, ${ }^{\text {a) }}$ B. J. Rodriguez, A. I. Kingon, and R. J. Nemanich \\ North Carolina State University, Raleigh, North Carolina 27695-7920 \\ J. S. Cross and M. Tsukada \\ Fujitsu Laboratories, Atsugi 243-0197, Japan
}

(Received 10 December 2002; accepted 1 March 2003)

\begin{abstract}
Piezoresponse force microscopy has been used to perform nanoscale characterization of the spatial variations in the imprint and switching behavior of (111)-oriented $\mathrm{Pb}(\mathrm{Zr}, \mathrm{Ti}) \mathrm{O}_{3}$-based capacitors on Pt electrodes. Mapping of polarization distribution in the poled capacitors as well as local $d_{33}-V$ loop measurements revealed a significant difference in imprint and switching behavior between the peripheral and inner parts of the capacitors. It has been found that the inner regions of the capacitors are negatively imprinted (with the preferential direction of the normal component of polarization upward) and tend to switch back after application of the positive poling voltage. On the other hand, switchable regions at the edge of the integrated capacitors generally exhibit more symmetric hysteresis behavior. Application of an ac switching voltage, contrary to what was expected, resulted in an increase of the negatively imprinted regions. The observed effect has been explained by incomplete or asymmetric switching due to the mechanical stress conditions existing in the central parts of the capacitors. (C) 2003 American Institute of Physics. [DOI: 10.1063/1.1570942]
\end{abstract}

Major development efforts in the field of ferroelectric thin films are currently focused on achieving high-density nonvolatile memories for mainstream microelectronic applications. ${ }^{1}$ There are a number of obstacles being faced. In a high-density ferroelectric random access memory (FRAM) device, a capacitor has to be scaled to lateral and thickness dimensions that may approach the stability limits for the ferroelectric phase. The intrinsic capacitor-tocapacitor variability becomes an issue as the number of anisotropic grains per capacitor becomes small. Microscopic reliability and failure mechanisms are a concern and need to be better understood. One of the major reliability problems of the ferroelectric memory devices is imprint, which can be defined as a preference of one polarization state over the opposite one. The most important manifestations of imprint are a shift of a hysteresis loop along the voltage axis and the instability of one of the polarization states, which may lead to a progressive loss of remnant polarization (retention loss).

Imprint behavior in ferroelectric thin film capacitors has been the subject of numerous studies by conventional macroscopic techniques..$^{2-5}$ However, only application of piezoresponse force microscopy (PFM) has provided a nanoscale insight into the scaling and intrinsic variability issues..$^{6-11}$ Recent PFM measurements revealed a significant size effect on hysteresis behavior and spatial variations in the ferroelectric properties across micro- and nanoscale capacitors. ${ }^{12-14}$ These variations can be attributed to the inherent inhomogeneity of the ferroelectric layer, to the effect of the constrained geometry of capacitors with high aspect ratio, or to the effect of capacitor processing damage. Generally, all these factors can contribute to the switching behavior and delineating the role of each factor is a very important issue.

${ }^{a)}$ Electronic mail: alexei_gruverman@ncsu.edu
In this letter, we present the results of PFM characterization of spatial variations in the ferroelectric properties of $\mathrm{Pb}(\mathrm{Zr}, \mathrm{Ti}) \mathrm{O}_{3}$ (PZT) FRAM capacitors. It has been shown that the switching and imprint behavior is a complex combination of electrical and mechanical effects.

The (111)-oriented Ca-, Sr-, and La-doped PZT layers with $\mathrm{Zr} / \mathrm{Ti}$ ratio of $40 / 60$ have been sputtered on the Pt bottom electrode. The thickness of the PZT layer was $200 \mathrm{~nm}$. Reactive ion etching has been used to fabricate 1 $\times 1.5-\mu \mathrm{m}^{2}$ capacitors with 50 -nm-thick $\mathrm{IrO}_{2}$ top electrodes on the PZT surface. The PZT layer has a (111) orientation and columnar grain structure with grain sizes on the order of $200 \mathrm{~nm}$ in diameter. Domain patterns have been visualized using the PFM imaging mode. ${ }^{6-8}$ In this study, visualization of domain patterns has been performed in individual capacitors by applying a modulation (imaging) voltage to the top electrode using a conductive PFM tip. The same tip was used to detect the mechanical displacement of the surface due to the converse piezoelectric effect. The top electrode did not conceal the domain structure, which has been imaged with a lateral resolution of less than $30 \mathrm{~nm}$. This approach ${ }^{15}$ allows one to circumvent the problem of an inhomogeneous field distribution generated by the probing tip in a ferroelectric film without a top electrode and to exclude possible contribution of Maxwell stress to the piezoresponse signal. ${ }^{16}$ During imaging, the film was scanned with an oscillating tip bias of $0.8 \mathrm{~V} \mathrm{rms}$ at $10 \mathrm{kHz}$. Local hysteresis loop have been measured by positioning the PFM tip at various sites on the top electrode and by measuring the PFM signal as a function of a dc voltage superimposed on the imaging voltage.

Figures 1(a) and 1(b) show PFM amplitude and phase images, respectively, of the array of as-grown $1 \times 1.5-\mu \mathrm{m}^{2}$ capacitors. Dark regions in the phase image [Fig. 1(b)] represent domains with the normal component of polarization oriented upward, and light regions represent a downward ori- 


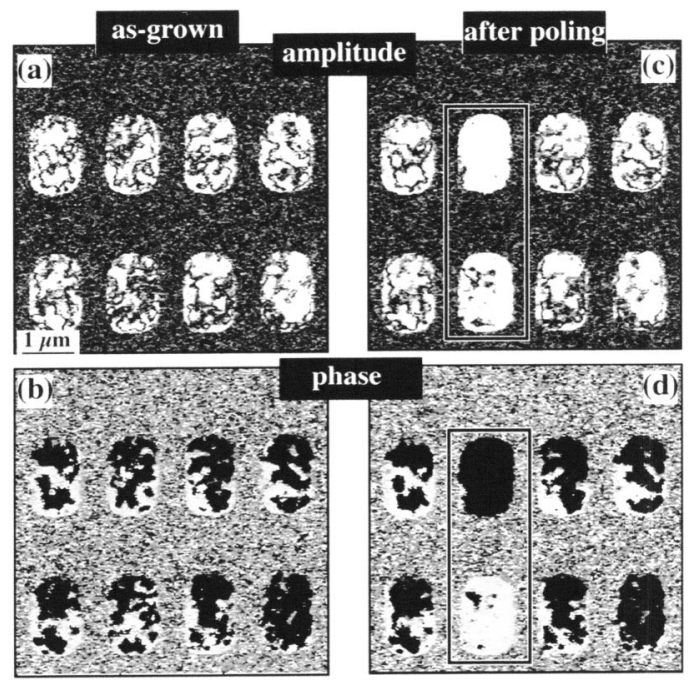

FIG. 1. PFM images of eight as-grown PZT capacitors before (a), (b) and after (c), (d) voltage pulses of opposite polarities have been applied to two of the capacitors (marked by a block). The upper of the two capacitors has been poled by the $-5 \mathrm{~V}, 3 \mathrm{~s}$ pulse, the lower one has been poled by the +5 V, 3 s pulse. Poling voltage was applied to the top electrode.

entation. Hereafter, dark regions in the phase image will be referred to as "negative" domains and bright regions as "positive" domains. In the amplitude image [Fig. 1(a)], domain boundaries appear as dark lines, indicating a weak piezoresponse signal.

The polarization distribution in Fig. 1(b) suggests that there is no initial preference in polarization direction in the as-processed capacitors. However, poling experiments revealed an asymmetry in switching patterns of the capacitors. In Figs. 1(c) and 1(d), two capacitors have been poled by applying voltage pulses of the same amplitude and duration $(5 \mathrm{~V}, 3 \mathrm{~s})$ but of opposite polarity. It can be seen that a capacitor poled by the $-5 \mathrm{~V}$ pulse exhibits uniform phase and amplitude contrast, indicating complete switching to a stable negative polarization state. In contrast, a capacitor poled by the $+5 \mathrm{~V}$ voltage exhibits nonuniform contrast suggesting that the remained dark regions either have not been switched under the applied voltage or switched back to the original polarization state after the voltage was turned off. This asymmetry in switching behavior can be an indication of negative imprint in the inner regions of the capacitor.

Local hysteresis loop measurements performed at different locations on the top electrode of one of the capacitors support this assumption. It has been found that inner regions, which remained dark after $+5 \mathrm{~V}$ poling, exhibit strongly asymmetric loops characterized by a shift toward positive voltages and suppressed positive switchable polarization [Fig. 2(a)]. Therefore, PFM hysteresis loop data, in agreement with the PFM imaging data, also suggest strong negative imprint in the inner regions of the capacitors. On the other hand, switchable regions at the edge of the FRAM capacitors generally exhibit more symmetric hysteresis behavior [Fig. 2(b)].

It has been shown previously that imprint can be explained in terms of the formation of an internal field of the space charges which stabilize the existing polarization state. ${ }^{17}$ A space-charge field can lead to a voltage shift of a hysteresis loop and to poor retention. ${ }^{17}$ A common procedure
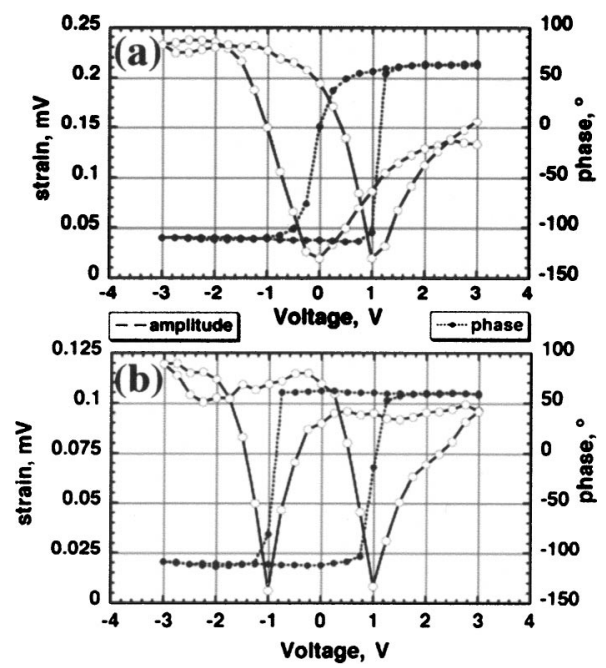

FIG. 2. Local piezoelectric hysteresis loops measured in the central (a) and peripheral (b) parts of a $1 \times 1.5-\mu \mathrm{m}^{2} \mathrm{PZT}$ capacitor.

for rejuvenation of imprinted capacitors is ac training: application of an ac voltage with amplitude higher than the coercive voltage for a short period of time (generally $<10^{5}$ cycles). In an ideal case, the ac training should lead to cyclic polarization reversal in the whole capacitor and, as a result, to the relaxation of space charges.

The effect of the ac training on imprint behavior was studied by applying an ac voltage $(4 \mathrm{Vrms}, 500 \mathrm{~Hz})$ to four FRAM capacitors for $5 \mathrm{~min}$ and then poling these capacitors by the $+5 \mathrm{~V}, 3 \mathrm{~s}$ voltage pulses. (The amplitude of the ac voltage has been chosen so that it will be higher than the coercive voltage determined from the polarization hysteresis loop). For control, four as-grown capacitors have been poled using voltage pulses of the same parameters without ac training. Results presented in Fig. 3 show that, contrary to what was expected, the ac training resulted in even less effective poling keep: much larger regions of the capacitors exhibit dark contrast after $+5 \mathrm{~V}$ poling; that is, show signs of negative imprint.

To check if the dark regions in Fig. 3 are due to pinned (nonswitchable) domains or due to domains formed as a result of backswitching, imaging of the capacitors has been performed with a dc bias of $+3 \mathrm{~V}$ superimposed on the ac modulation voltage. In this case, the phase and amplitude contrast become uniform in all capacitors indicating complete polarization reversal under the dc bias (Fig. 4). However, subsequent conventional PFM imaging (using the ac modulation voltage only) revealed almost the same domain pattern as before the ac/dc imaging. These results support the assumption that the dark regions seen in the positively poled capacitors in Fig. 3 are domains formed as a result of backswitching and not pinning. This observation again suggests strong negative imprint in the inner regions of the capacitors.

The observed degradation of poling efficiency of the FRAM capacitors as a result of the ac training can be explained if we assume that, for some reasons, which will be discussed later, the ac training voltage does not induce complete polarization reversal in some regions with a negative polarization state. Then injection and interface entrapment of additional electronic charges would further imprint the negative polarization. In principle, the same effect could take 


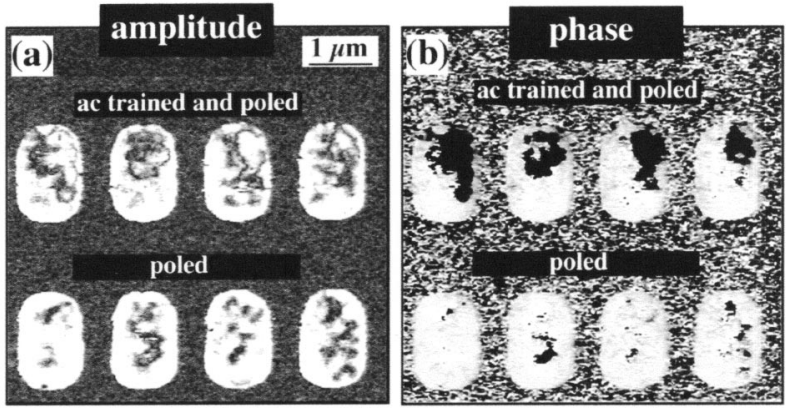

FIG. 3. PFM images illustrating a detrimental effect of the ac training on imprint behavior. Capacitors in the upper row have been trained for $5 \mathrm{~min}$ by an ac voltage ( $4 \mathrm{Vrms}, 1 \mathrm{kHz})$ and then poled by $+5-\mathrm{V}, 3-\mathrm{s}$ voltage pulses. For reference, as-grown capacitors in the bottom row have been also poled by $+5-\mathrm{V}, 3-\mathrm{s}$ voltage pulses.

place if the switching time to a negative state was much shorter than to a positive state. ${ }^{18,19}$ In this case, the total time of the capacitor in the negative polarization state during training will be much larger than that for the positive state. This will induce further screening of the negative polarization and will lead to stronger negative imprint.

The model just described explains the worsening effect of the ac training on the imprint behavior of the FRAM capacitors. However, it does not explain the observed spatial inhomogeneity of the switching and imprint behavior. Recently, Stolichnov et al. ${ }^{14}$ reported an anomaly in the polarization distribution of $2 \times 3-\mu \mathrm{m}^{2}$ (111)-oriented PZT capacitors. This result, which is explained in terms of straininduced phase transition, strongly suggests a mechanical stress effect on imprint and switching behavior of the (111)oriented PZT capacitors. It should be noted that the capacitors studied in Ref. 14 are similar in structure, but different in thickness and size to those being investigated in the present study. The apparent difference between the switching patterns observed in our study and in Ref. 14 can be attributed to a strong size effect of polarization distribution. It can be speculated that in our case, the misfit strain is not sufficient to induce polarization reversal in the central regions. Instead, it creates preferential conditions for the negative polarization state. The central regions of the PZT capacitors are likely under tensile stress, which can affect the local coercive voltage by the stress-induced phase transition. Experimental observation of such effect was recently made by Kelman. ${ }^{20}$ It is likely then, that tensile stress causes incomplete switching in the central regions during the ac training, which eventually leads to stronger imprint as discussed earlier.

In summary, PFM mapping of polarization distribution as well as local $d_{33}-V$ loop measurements revealed significant variations in imprint and switching behavior within integrated PZT capacitors. The inner regions of the capacitors exhibit a strong tendency to negative imprint. The obtained results suggest that these variations are a result of a complex combination of mechanical stress and electric-field effects. The contribution of each effect may vary drastically not only with the change in the capacitor size but also with the change in the processing conditions. Detailed analysis of symmetry change and polarization map as a function of the stress con-
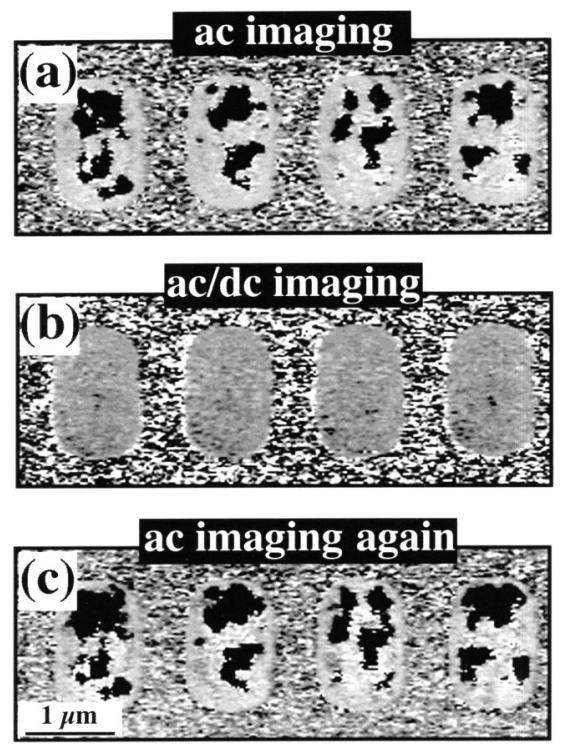

FIG. 4. PFM phase images illustrating a backswitching effect. (a) Conventional (ac voltage only) PFM imaging of ac trained capacitors. (b) The same capacitors imaged with ac/dc bias applied to the tip (dc bias of $+3 \mathrm{~V}$ ). (c) The same capacitors visualized again using conventional PFM imaging.

ditions and the depolarization field is required to better understand the size dependence of the imprint and switching behavior of the integrated FRAM capacitors.

The authors acknowledge financial support of Fujitsu Ltd. throughout this project.

${ }^{1}$ O. Auciello, J. F. Scott, and R. Ramesh, Phys. Today 1998, 22.

${ }^{2}$ D. Dimos, W. L. Warren, M. Sinclair, B. A. Tuttle, and R. W. Schwartz, J. Appl. Phys. 76, 4305 (1994).

${ }^{3}$ G. Arlt and H. Neumann, Ferroelectrics 87, 109 (1988).

${ }^{4}$ A. Kholkin, V. Yarmarkin, B. Goltsman, and J. Baptista, Integr. Ferroelectr. 35, 261 (2001).

${ }^{5}$ M. Grossmann, O. Lohse, D. Bolten, U. Boettger, R. Waser, W. Hartner, M. Kastner, and G. Schindler, Appl. Phys. Lett. 76, 363 (2000).

${ }^{6}$ A. Gruverman, O. Auciello, and H. Tokumoto, Annu. Rev. Mater. Sci. 28, 101 (1998).

${ }^{7}$ T. Hidaka, T. Maruyama, I. Sakai, M. Saitoh, L. A. Wills, R. Hiskes, S. A. Dicarolis, and J. Amano, Integr. Ferroelectr. 17, 319 (1997).

${ }^{8}$ S. Hong, J. Woo, H. Shin, J. Jeon, Y. E. Pak, E. L. Colla, N. Setter, E. Kim, and K. No, J. Appl. Phys. 89, 1377 (2001).

${ }^{9}$ J. A. Christman, S.-H. Kim, H. Maiwa, J.-P. Maria, B. J. Rodriguez, A. I. Kingon, and R. J. Nemanich, J. Appl. Phys. 87, 8031 (2000).

${ }^{10}$ S. Buhlmann, B. Dwir, J. Baborowski, and P. Muralt, Appl. Phys. Lett. 80, 3195 (2002).

${ }^{11}$ C. S. Ganpule, A. Stanishevsky, S. Aggarwal, J. Melngailis, E. Williams, R. Ramesh, V. Joshi, and C. A. Paz de Araujo, Appl. Phys. Lett. 75, 3874 (1999).

${ }^{12}$ M. Alexe, A. Gruverman, C. Harnagea, N. D. Zakharov, A. Pignolet, D. Hesse, and J. F. Scott, Appl. Phys. Lett. 75, 1158 (1999).

${ }^{13}$ M. Alexe, C. Harnagea, D. Hesse, and U. Gösele, Appl. Phys. Lett. 79, 242 (2001).

${ }^{14}$ I. Stolichnov, E. Colla, A. Tagantsev, S. Bharadwaja, S. Hong, N. Setter, J. S. Cross, and M. Tsukada, Appl. Phys. Lett. 80, 4804 (2002).

${ }^{15}$ O. Auciello, A. Gruverman, and H. Tokumoto, Integr. Ferroelectr. 15, 107 (1997).

${ }^{16}$ S. V. Kalinin and D. A. Bonnell, Phys. Rev. B 65, 125408 (2002).

${ }^{17}$ R. R. Mehta, B. D. Silverman, and J. T. Jacobs, J. Appl. Phys. 44, 3379 (1973).

${ }^{18}$ X. Lu, J. Zhu, X. Zhang, Z. Liu, Y. Wang, and X. Chen, Appl. Phys. Lett. 80, 2961 (2002).

${ }^{19}$ V. Ya. Shur, E. L. Rumyantsev, E. V. Nikolaeva, E. I. Shishkin, and I. S. Baturin, J. Appl. Phys. 90, 6312 (2001).

${ }^{20}$ M. B. Kelman, L. F. Schloss, P. C. McIntyre, B. C. Hendrix, S. M. Bilodeau, and J. F. Roeder, Appl. Phys. Lett. 80, 1258 (2002). 